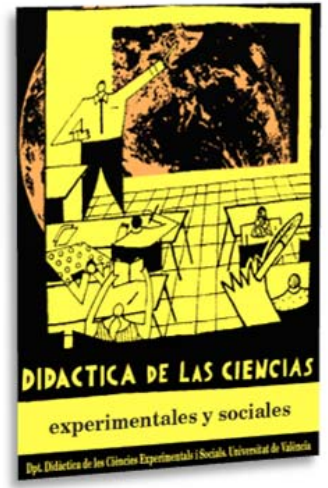

\title{
¿Son los huertos escolares en educación infantil una realidad o una innovación educativa? Estudio de centros escolares de la ciudad de Córdoba (España) y propuestas de cambio desde la Universidad
}

\author{
Are school gardens in early childhood education a \\ reality or an educational innovation? Study of \\ schools in the city of Córdoba (Spain) and \\ proposals for change from the University
}

DOI: $10.7203 / D C E S .36 .12535$

Jorge Alcántara Manzanares

Universidad de Córdoba, b62almaj@uco.es ORCID iD: http://orcid.org/0000-0003-2482-1615

\section{Manuel Mora}

Universidad de Córdoba, q82momam@uco.es ORCID iD: http://orcid.org/0000-0002-4052-9828

José Carlos Arrebola

Universidad de Córdoba, q92arhaj@uco.es

ORCID iD: http://orcid.org/0000-0001-9337-6207

\begin{abstract}
Jerónimo Torres-Porras
Universidad de Córdoba, jeronimo.torres@uco.es ORCID iD: http://orcid.org/0000-0003-1900-7870
\end{abstract}

\section{Sebastián J. Rubio}

Universidad de Córdoba, sjrubio@uco.es

ORCID iD: http://orcid.org/0000-0002-2537-723X

\author{
Lidia Rodríguez Rodríguez \\ Universidad de Córdoba \\ lidiarodriguez-1991@hotmail.com
}

\begin{abstract}
RESUMEN: El presente artículo pretende vislumbrar si los centros de educación infantil de la ciudad de Córdoba tienen huerto escolar, las razones por las que carecen en su caso, y si conocen las técnicas de planificación, diseño e implementación. Para analizar la existencia del huerto se realizaron 93 encuestas telefónicas, obteniéndose que más de un $50 \%$ de los centros no tenían, ya sea por falta de espacio, por no habérselo planteado o por falta de conocimientos. Casi el $70 \%$ de los centros sin huerto alegaron no conocer las técnicas. Con estos resultados, queda justificado el objetivo de formar al alumnado de Grado en Educación Infantil sobre este recurso. En este caso, la iniciativa educativa presentada consistió en la preparación de huertos portátiles o reproducibles en cualquier espacio exterior.
\end{abstract}

Palabras CLAVE: Huerto Escolar, Ecohuerto, Educación Infantil, Educación Ambiental, Desarrollo Sostenible.

ABSTRACT: The present article aims to assess if early childhood education schools of the city of Córdoba have a school garden, the reasons why they have not (in its case), and if they know the planning design and implementation techniques. To analyse the existence of school gardens, 93 telephone surveys were carried out. Results show that more than $50 \%$ of schools have no gardens, as a result of the lack of space, of knowledge or just for not having thought of that possibility. Almost $70 \%$ of the schools without gardens claimed not to know the techniques. Thus, the objective of training the students of the Bachelor Degree in Early Childhood Education is more than justified. In this case, the educational initiative presented consisted of the preparation of portable or reproducible orchards in any outdoor space.

KEYworDs: School Garden, Organic Orchard, Early Childhood Education, Environmental Education, Sustainable Development.

Fecha de recepción: octubre de 2018

Fecha de aceptación: febrero de 2019

En primer lugar, se ha de agradecer a la Facultad de Ciencias de la Educación de la Universidad de Córdoba el apoyo y el interés mostrado en las iniciativas relativas al huerto aquí presentadas. También, al alumnado de los grados de Educación Infantil y Educación Primaria por su participación en las prácticas. Por último, a la Universidad de Córdoba por la financiación otorgada para la instalación del huerto escolar no permanente y el invernadero gracias a la concesión del Proyecto Coordinado de Innovación de la Facultad de Ciencias de la Educación de la Universidad de Córdoba (convocatoria 2015 y código 2015-1-6001). 


\section{INTRODUCCIÓN}

La sociedad actual es cada vez más urbanita y está más desconectada de la naturaleza, lo que puede repercutir de forma negativa en el estado físico y mental de niños y niñas, y reducir su afinidad hacia la naturaleza (Ozdemir y Yilmaz, 2008; Wells 2000; Collado y Corraliza, 2011). El contacto con espacios naturales es positivo para el desarrollo infantil (Wells, 2000; Faber, Taylor y Kuo, 2009; Wolch et al., 2011; Corraliza, Collado y Bethelmy, 2012), además de que puede motivar un mayor respeto hacia la naturaleza (Davis, Rea y Waite, 2006; Chawla y Cushing, 2007).

El huerto crea una conexión entre la naturaleza y los pequeños y pequeñas, donde pueden observar, por ejemplo, el ciclo de vida de las plantas, favoreciendo que las entiendan como seres vivos. El huerto permite al alumnado sembrar, cultivar y recolectar. De este modo, a través de la observación y manipulación podrán comprender el ciclo de nacimiento, crecimiento, maduración y muerte de las plantas. Además, las niñas y niños podrán tomar conciencia de los cuidados que estos seres vivos necesitan para crecer y desarrollarse (Kauffman, 1995).

El huerto es un ecosistema complejo que permite introducir la educación ambiental en el aula de infantil, ya que a través de este los niños y las niñas, en primer lugar, analizarán e investigarán directamente el medio, en segundo lugar, conocerán los elementos que lo conforman, así como las interacciones y los cambios que se producen en él, y por último, también adquirirán valores y actitudes de respeto hacia el medio ambiente (Ceballos, 2017).

Por otra parte, en el ámbito de la educación superior, los huertos escolares se consideran una manera de introducir la sostenibilidad en el currículo universitario en los grados de educación, siendo estos auténticos vertebradores de cambio, si se establecen además relaciones participativas con la comunidad (Barrón Ruiz y Muñoz Rodríguez, 2015).

El huerto escolar es una fuente de alimentos saludables que posibilita al alumnado conocer de dónde proceden los alimentos, cómo se producen o qué beneficios tienen para la salud del ser humano (Ratcliffe, Merrigan, Rogers y Goldberg, 2011).

En suma, "hoy en día ya nadie pone en duda que el huerto es una herramienta educativa casi perfecta que hay que promocionar y potenciar” (Escutia, 2009, p. 20), con la que se aprenden aspectos de todas las áreas de conocimiento establecidas en el currículum de educación infantil, fomentando la adquisición de las competencias básicas (Botella, Hurtado y Cantó, 2014).

Debido a los beneficios que aporta el huerto escolar en el proceso de enseñanza-aprendizaje en educación infantil y por ejemplos exitosos de puesta en práctica en centros escolares que veremos a continuación, cabría pensar que estos ya no constituyen una innovación educativa, sino que son una realidad. A través de este trabajo, que tiene como ámbito de estudio los centros de educación infantil de la ciudad de Córdoba (España), se pretende confirmar o refutar dicha afirmación y proponer, en cualquier caso, herramientas de mejora, a través de la formación en el Grado de Educación Infantil.

\section{MARCO TEÓRICO}

La historia de la agricultura se puede decir que coincide con la historia de la civilización, aunque se cree que mucho antes de que aparecieran las primeras civilizaciones ya el hombre primitivo se encargaba de recoger las plantas provenientes del campo para poder alimentarse. Ahí, precisamente, se puede colocar el inicio de la agricultura. El ser humano cubría sus necesidades alimentarias a través de los alimentos que la tierra producía espontáneamente, pero más adelante, estos no eran suficientes. Así fue, que las personas aprendieron a cultivar por necesidad. A lo largo del tiempo las costumbres y conocimientos acerca del cultivo se fueron transmitiendo de unas civilizaciones a otras (Flórez, 2009).

En un principio, los agricultores y agricultoras rotaban sus cultivos, la tierra era fertilizada con productos orgánicos y sus cosechas eran puramente ecológicas. De este modo, la tierra era fértil 
por muchos años. Todo esto cambió cuando al iniciarse el s. XX llegó la agricultura industrializada, a la que le interesa obtener beneficios rápidos y en un corto plazo de tiempo, sin tener en cuenta las consecuencias para el abastecimiento de las futuras generaciones (Goodall, 2007).

La agricultura ecológica también recibe el nombre de agricultura orgánica o agricultura biológica. Según El-Hage y Hattam (2003): “el término agricultura orgánica se refiere al proceso que utiliza métodos que respetan el medio ambiente, desde las etapas de producción hasta las de manipulación y procesamiento” (p.3).

Remmers (1993) sostiene que la agricultura ecológica surgió como reacción a la agricultura dominante y al modelo de consumo que la sociedad desarrolla, y que intenta llevar a cabo alternativas ecológicas a la agricultura convencional e incluso busca mejorar el medio ambiente.

El aumento de la superficie ecológica en España y Andalucía ha sido rápido, siendo fundamental la subvención comunitaria europea. En la actualidad España se ha convertido en el país de la Unión Europea que más superficie de cultivo dedica a producción ecológica. Por su parte, Andalucía lidera el ranking español de superficie ecológica (Larrubia Vargas, Natera Ribas y Navarro Rodríguez, 2017).

La agricultura ecológica, frente a la agricultura convencional, mejora la biodiversidad y conlleva un menor desgaste ambiental, disminuyendo el uso de productos químicos, el gasto de energía y promoviendo el uso más eficiente del agua (De los Ríos-Carmenado, Becerril-Hernández y Rivera, 2016).

La alimentación es una necesidad básica que tiene el ser humano. Se produce cuando un organismo ingiere alimentos que proporcionan nutrientes necesarios para tener energía y poder desarrollarse correctamente. Izquierdo, Armenteros, Lancés y Martín (2004) van más allá y afirman: "La alimentación es una cadena de hechos que comienzan con el cultivo, selección y preparación del alimento hasta las formas de presentación y consumo de un grupo de ellos” (p.1).

Una adecuada alimentación corresponde con una dieta saludable, basada en alimentos variados y atendiendo a las diferentes etapas de vida, ya que dependiendo de la etapa en la cual nos encontremos, necesitaremos alimentarnos de una forma u otra. Una dieta saludable ayudará, a través de la nutrición, a que nuestro organismo funcione correctamente (Silva, 2007). Una buena educación alimentaria desde la infancia ayudará a prevenir enfermedades y problemas nutricionales, tales como, la obesidad, diabetes o trastornos cardiovasculares. Hidalgo y Güemes (2011) sostienen:

Durante la infancia y adolescencia, es necesario un equilibrado aporte nutricional y ejercicio físico para obtener un adecuado estado de salud, un óptimo crecimiento y desarrollo físico y psicosocial y ayudar al establecimiento de hábitos alimentarios saludables que permitan prevenir problemas de salud futuros. (p.352)

Hay algunos estudios que indican que los alimentos ecológicos son más nutritivos. Otros han investigado sobre los beneficios en la salud de las personas del consumo de productos ecológicos. Pero de momento no hay evidencias científicas para concluir con rotundidad que los alimentos ecológicos son más saludables (Brantsæter, Ydersbond, Hoppin, Haugen y Meltzer, 2017).

Un factor importante a la hora de proporcionar una buena educación alimentaria a los niños y las niñas es conocer los distintos alimentos, que se dividen en origen vegetal (frutas, verduras, cereales) y origen animal (carne, pescado, huevos, leche), así como sus funciones nutritivas para el organismo. Las personas cercanas a los pequeños y las pequeñas, principalmente la familia y la escuela, son decisivas en su educación alimentaria. Según Miralpeix (2003), para que los niños y niñas tengan una buena dieta, los adultos que los rodean igualmente deberán tenerla. Una buena educación de hábitos por parte del entorno permitirá que los pequeños y pequeñas coman bien cuando se introduzcan en la alimentación adulta.

Así, el comedor escolar es un servicio que ofrecen los centros educativos al alumnado, su función principal es la de aportar una buena alimentación y nutrición a estos, así como, desempeñar una función educativa y social (Aranceta, 2008). En aquellos centros que disponen de cocina, 
huerto escolar y los permisos necesarios de la administración, muchos de los platos que se preparan en el comedor pueden ser elaborados con los alimentos del huerto escolar. El propio alumnado se puede encargar de ello con la ayuda de la familia y los maestros y maestras. De este modo, muchos niños y niñas se animarán a probar sabores nuevos, e incluso a comer las verduras y hortalizas a las que suelen ser reacios, ya que tendrán la motivación de consumir las verduras, hortalizas y frutas que han cultivado y recolectado con sus propias manos, que además serán ecológicos.

Se puede entender por "huerto" cualquier espacio de tierra disponible, el cual está destinado al cultivo de verduras, legumbres, hortalizas y árboles frutales. Escutia (2009) sostiene que el huerto es el lazo que une al ser humano con la tierra, siendo un símbolo de salud, intercambio, autosuficiencia, humanidad y tradición, que permite a todos y a todas ser agricultores y agricultoras y obtener alimentos sin tener que requerir del sistema monetario.

El huerto ecológico es aquel que se rige por los criterios de la agricultura ecológica, ya que, como nos dice Bueno (2010), la preocupación por la alimentación y la salud en la población ha crecido, cada vez son más los agricultores y agricultoras que prefieren cultivar sus propios alimentos e intentan prescindir de los productos químicos, y siguen los principios de la agricultura ecológica llevando a cabo métodos naturales y respetuosos con el medio ambiente y la salud.

Cuando el huerto está dentro de la escuela, se denomina huerto escolar. Para que el huerto escolar sea exitoso, éste debe ser simple, el profesorado o el personal encargado del huerto tiene que adaptarse y hacer frente a los cambios. Hay que saber escoger las plantas adecuadas, ya que algunas son más sencillas de cultivar. El huerto debe ser ecológico porque hay que enseñar a los niños y niñas a ser respetuosos con el medio ambiente, así como ser tolerantes y pacientes, pero lo más importante es divertirse cultivando (Escutia, 2009).

Como bien explica Muelhoff (2006), los huertos escolares son laboratorios al aire libre y constituyen una oportunidad para la implicación de las familias en la escuela.

Una relación de los objetivos generales para la etapa de educación infantil, recogidos en la Ley Orgánica 8/2013 para la Mejora de la Calidad Educativa (LOMCE), que se pueden trabajar con el desarrollo de un huerto son los mostrados en la Tabla 1.

TABLA 1. Relación objetivos generales currículo y objetivos didácticos del uso del huerto escolar

\begin{tabular}{|c|c|}
\hline Objetivos currículo & ticos \\
\hline \multirow{2}{*}{$\begin{array}{l}\text { Adquirir autonomía en la realización } \\
\text { de sus actividades habituales y en la } \\
\text { práctica de hábitos básicos de salud y } \\
\text { bienestar y desarrollar su capacidad de } \\
\text { iniciativa. }\end{array}$} & $\begin{array}{l}\text { 1. Trabajar con los alimentos de una } \\
\text { forma ecológica y saludable, manipularlos y } \\
\text { conocer información acerca de ellos. }\end{array}$ \\
\hline & $\begin{array}{l}\text { 2. Fomentar en los niños y las niñas un } \\
\text { mayor interés hacia los productos saludables. }\end{array}$ \\
\hline $\begin{array}{l}\text { Establecer relaciones sociales } \\
\text { satisfactorias en ámbitos cada vez más } \\
\text { amplios, teniendo en cuenta las emociones, } \\
\text { sentimientos y puntos de vista de los demás, } \\
\text { así como adquirir gradualmente pautas de } \\
\text { convivencia y estrategias en la resolución } \\
\text { pacífica de conflictos. }\end{array}$ & $\begin{array}{l}\text { 3. Cubrir necesidades básicas de } \\
\text { socialización, aspecto que será fundamental } \\
\text { para el correcto desarrollo tanto emocional, } \\
\text { físico, psicológico, social y educativo, gracias } \\
\text { a que los alumnos y alumnas se sienten } \\
\text { pertenecientes a un grupo que tiene un fin } \\
\text { común y que trabaja de forma unida para } \\
\text { alcanzarlo }\end{array}$ \\
\hline $\begin{array}{l}\text { Observar y explorar su entorno } \\
\text { físico, natural, social y cultural, generando } \\
\text { interpretaciones de algunos fenómenos y } \\
\text { hechos significativos para conocer y } \\
\text { comprender la realidad y participar en ella } \\
\text { de forma crítica. }\end{array}$ & $\begin{array}{l}\text { 4. Analizar la realidad a través de la } \\
\text { observación y entender ciertos fenómenos, } \\
\text { tanto naturales (crece una planta o un fruto), } \\
\text { sociales (interacción entre compañeros, } \\
\text { división del trabajo, cooperación) e incluso } \\
\text { económicos (trueque). }\end{array}$ \\
\hline
\end{tabular}




\begin{tabular}{|c|c|}
\hline & \\
\hline \multirow[t]{2}{*}{$\begin{array}{l}\text { Comprender y representar algunas } \\
\text { nociones y relaciones lógicas y } \\
\text { matemáticas referidas a situaciones de la } \\
\text { vida cotidiana, acercándose a estrategias } \\
\text { de resolución de problemas. }\end{array}$} & $\begin{array}{l}\text { 5. Trabajar el tiempo y el espacio a } \\
\text { través de conceptos como “encima-debajo" (de } \\
\text { la tierra / superficie), derecha-izquierda, } \\
\text { conceptos temporales (cada cuantos días } \\
\text { regar). }\end{array}$ \\
\hline & $\begin{array}{l}\text { 6. Aprender tamaños, formas y } \\
\text { conceptos lógico-matemáticos a través de la } \\
\text { manipulación. }\end{array}$ \\
\hline $\begin{array}{l}\text { Aproximarse a la lectura y escritura } \\
\text { en situaciones de la vida cotidiana a través } \\
\text { de textos relacionados con la vida } \\
\text { cotidiana, valorando el lenguaje escrito } \\
\text { como instrumento de comunicación, } \\
\text { representación y disfrute. }\end{array}$ & $\begin{array}{l}\text { 7. Identificar las frutas, hortalizas y } \\
\text { especies aromáticas, leer su nombre y saber } \\
\text { escribirlo para desarrollar la lectoescritura en } \\
\text { el alumnado. }\end{array}$ \\
\hline $\begin{array}{l}\text { Conocer y participar en algunas } \\
\text { manifestaciones culturales y artísticas de } \\
\text { su entorno, teniendo en cuenta su } \\
\text { diversidad y desarrollando actitudes de } \\
\text { interés, aprecio y respeto hacia la cultura } \\
\text { andaluza y la pluralidad cultural. }\end{array}$ & $\begin{array}{l}\text { 8. Conocer la gastronomía local como } \\
\text { elemento cultural de especial relevancia, } \\
\text { aprendiendo a preparar recetas típicas } \\
\text { mediante los productos del huerto y con la } \\
\text { ayuda de las familias. }\end{array}$ \\
\hline
\end{tabular}

Fuente: Elaboración propia a partir de la LOMCE

Muchos son ya los centros educativos que se han animado a crear con su alumnado huertos ecológicos como proyecto educativo; entre ellos destaca la Red de Ecohuertos escolares, un proyecto que se inicia a partir del Plan Columela de Agricultura ecológica, proporcionando formación y asesoramiento al profesorado, así como, materiales didácticos y actividades complementarias de sensibilización. Actualmente llevan a cabo la creación de huertos ecológicos en nueve centros de diversos municipios de la provincia de Sevilla (España): Mairena del Alcor, El Viso del Alcor, Carmona, La Campana, Fuentes de Andalucía, La Luisiana y Écija.

Los niños y niñas de estos centros en primer lugar, preparan el terreno, incidiendo en la importancia que tiene el suelo en la agricultura ecológica y en segundo lugar, se ocupan del cultivo recogiendo su seguimiento y sus impresiones en un "cuaderno viajero", realizan concursos de huertos..., entre otras actividades y juegos. Entre sus objetivos destacan algunos como sensibilizar al alumnado acerca de los beneficios de los alimentos ecológicos, educar en valores ambientales a las niñas y niños y ver la agricultura ecológica como un proyecto que apueste por el desarrollo sostenible (Grupo de Desarrollo Rural de la Campiña y los Alcores, s.f.).

Otra experiencia de gran interés, en este caso para comprender la importancia de los huertos como agentes de cambio social, es la desarrollada en el CEIP Olivar de Quinto. Lo que comenzó como una actividad educativa momentánea, ha ido involucrando a toda la comunidad educativa, para después convertirse en un elemento dinamizador del tejido social del barrio de Montequinto (Dos Hermananas, Sevilla), por medio de huertos urbanos basados en la agricultura ecológica (Rodríguez-Marín, Fernández-Arroyo y García Díaz, 2015).

\section{OBJetivos}

El objetivo general de este trabajo es responder a la pregunta: ¿Son los huertos escolares una realidad o una innovación educativa? Para responder a la misma se ha tomado como ámbito de estudio la ciudad de Córdoba (España) y como marco educativo a aquellos centros en los que haya educación infantil. En este sentido, se han consignado los siguientes objetivos concretos: 
- Analizar si el huerto escolar está presente en los centros de educación infantil de la ciudad de Córdoba.

- Identificar las razones por las que no está presente el huerto escolar en aquellos centros de educación infantil que carezcan del mismo.

- Analizar si en dichos centros se conocen algunas de las técnicas de planificación, diseño e implementación de un huerto escolar.

Tras los resultados obtenidos en la investigación, se propone un objetivo para ayudar a aumentar la cantidad de centros escolares con huerto mediante la inclusión de esta temática en la formación inicial de formadoras y formadores de educación infantil:

- Mostrar diferentes técnicas de cultivo que posibilitan el desarrollo de esta actividad en cualquier centro a través de las prácticas llevadas a cabo en la Facultad de Ciencias de la Educación de la Universidad de Córdoba como parte de la formación del alumnado de Grado en Educación Infantil.

\section{Metodología}

Para dar respuesta a los tres primeros objetivos anteriormente expuestos, se ha desarrollado una investigación mixta en la que se han realizado encuestas telefónicas a colegios, tanto públicos como privados, en dos fases:

a) En una primera fase, se ha pretendido contestar a la pregunta: ¿Cuántos centros de educación infantil de la ciudad de Córdoba (España) tienen huerto escolar? Para responder a la misma se ha intentado contactar telefónicamente con los 106 centros de educación infantil incluidos en el listado telefónico páginas amarillas, habiendo obtenido respuesta de 93 centros, 37 públicos y 56 privados. Se ha puesto de manifiesto que se estaba realizando una investigación sobre huertos escolares y se ha preguntado si en su centro disponían de huerto escolar. Teniendo en cuenta que, según los datos arrojados por el buscador de centros de la Consejería de Educación de la Junta de Andalucía, el número de centros que cubre la etapa de educación infantil asciende en la localidad de Córdoba a 226, y habiendo contactado con 93 centros, el tamaño de la muestra presenta un intervalo de confianza del $95 \%$ y un margen de error inferior al $8 \%$.

b) La segunda fase se ha centrado en aquellas escuelas que habían manifestado no tener huerto escolar. Tras contactar con la persona responsable se realizaron las siguientes preguntas:

P.1. ¿Cuáles son las causas por las que no disponen de huerto escolar en su centro?

P.2. ¿De qué espacios disponen?

P.3. ¿Conoce las distintas técnicas para instaurar un huerto en su centro?

Las respuestas se han transcrito y se han analizado buscando patrones de respuesta y estableciendo categorías a posteriori. Más tarde se ha realizado un análisis de frecuencia de dichas categorías.

El cuarto objetivo es mostrar al alumnado de Grado en Educación Infantil diferentes técnicas que posibilitan instaurar un huerto en cualquier centro a través de las prácticas llevadas a cabo en la Facultad de Ciencias de la Educación de la Universidad de Córdoba como parte de su formación, que son: 
1. El huerto escolar no permanente con fines docentes generado gracias al Proyecto Coordinado de Innovación Educativa de la Facultad de Ciencias de la Educación, durante el curso 2015/2016.

2. Las cajas de siembra y cultivo como trabajo de prácticas de la asignatura Salud y Consumo, optativa de cuarto curso de Grado en Educación Infantil.

\section{RESULTADOS Y DISCUSIÓN}

A continuación se muestran los resultados estructurados en cuatro epígrafes dando respuesta a cada uno de los objetivos propuestos en este trabajo.

\subsection{Analizar si el huerto escolar está presente en los centros de educación infantil de la ciudad de Córdoba}

Los resultados de la primera fase relativos a la pregunta “¿Cuántos centros de educación infantil de la ciudad de Córdoba (España) tienen huerto escolar?” son:

- 36 de los 93 colegios (38,7 \%) de Córdoba tienen huerto en su centro y hacen uso de él con frecuencia. De dichos 36 centros escolares, 15 son privados/concertados y 21 son públicos.

- 2 de los 93 colegios (2,1 \%) de Córdoba, 1 privado/concertado y 1 público, tienen huerto en su centro pero lo utilizan ocasionalmente.

- 6 de los 93 colegios (6,4 \%) de Córdoba, en este caso todos públicos, tienen huerto en su centro pero no es funcional, es decir, no hacen uso de él durante este curso escolar.

- 1 de los 93 colegios, en este caso público, únicamente tiene árboles frutales.

- 48 de los 93 (51,6 \%) colegios, 20 privados/concertados y 28 públicos, no tienen huerto en su centro.

Más del 50 por ciento de los centros educativos encuestados donde se imparte educación infantil en la ciudad de Córdoba no tienen huerto escolar. Si obtenemos la frecuencia de los privados/concertados y de los públicos de manera independiente, el $57 \%$ de los centros públicos y el $57 \%$ de los privados/concertados no tienen huerto escolar. Por tanto, el huerto escolar no es una realidad educativa en más de la mitad de dichos centros. Dadas las ventajas de los huertos escolares, dicho porcentaje resulta elevado. Más aun cuando las condiciones ambientales de la ciudad de Córdoba son proclives a este tipo de prácticas educativas, como el clima mediterráneo con inviernos suaves y a la disponibilidad de agua de calidad gracias al abastecimiento municipal. Dichos resultados han dado todavía mayor relevancia a la segunda fase de la investigación que pretende escudriñar las razones que determinan la ausencia de huertos escolares en los centros educativos encuestados.

\subsection{Identificar las razones por las que no está presente el huerto escolar en aquellos centros de educación infantil que carezcan del mismo}

A continuación, se muestran los resultados de la segunda fase. En el Gráfico 1 se muestran los resultados relativos a la pregunta: ¿Cuáles son las causas por las que no disponen de huerto escolar en su centro? 
FigURA 1. Frecuencia relativa, en tanto por ciento, de respuesta de los centros escolares con educación infantil de la ciudad de Córdoba en relación a la pregunta: ¿Cuáles son las causas por las que no disponen de huerto escolar en su centro?

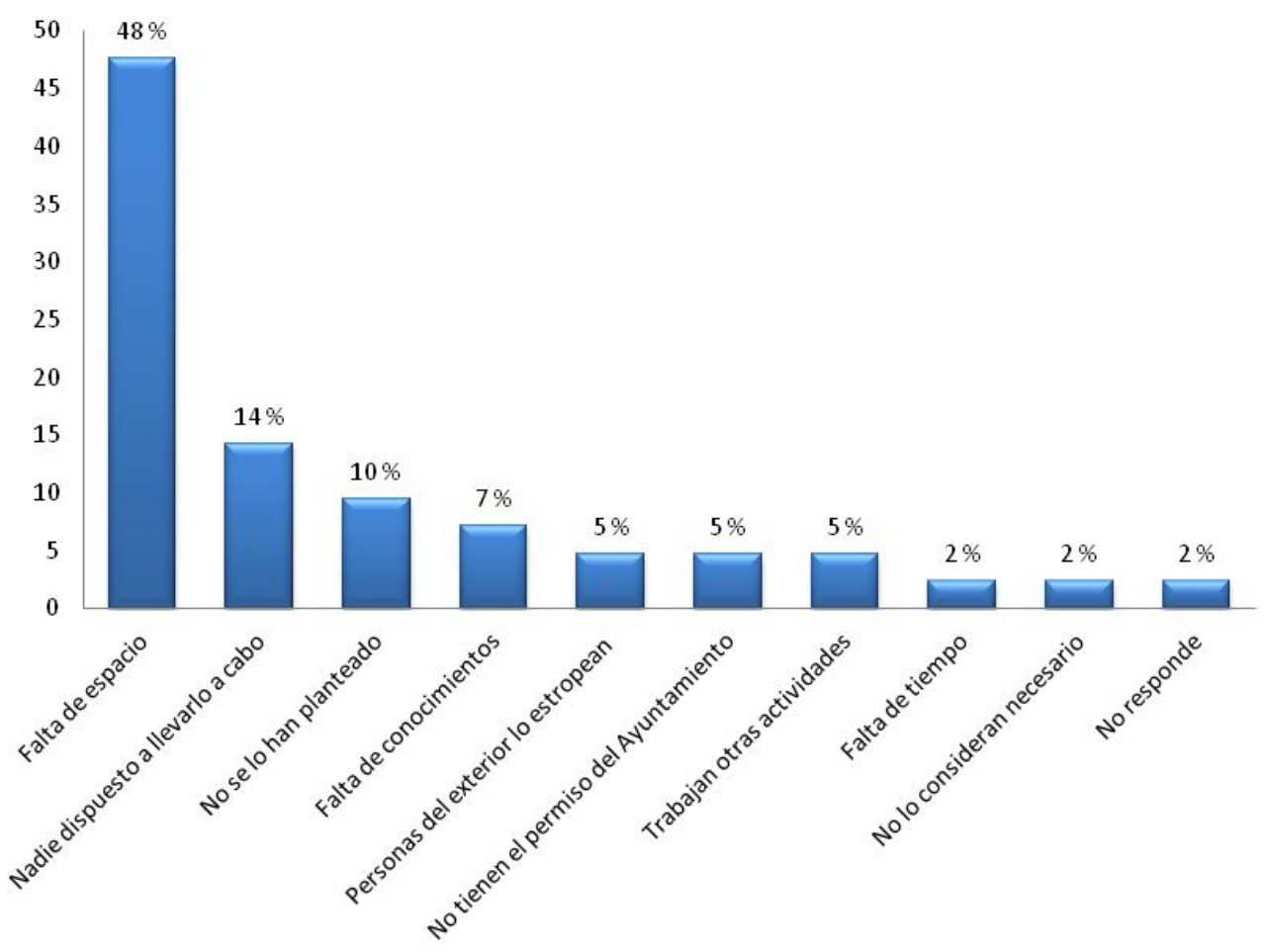

Fuente: Elaboración propia

Se han detectado 10 categorías, tras analizar las respuestas. En cuanto a la frecuencia relativa de dichas categorías, la mayor se corresponde con "Falta de Espacio" llegando está casi al 50 \%. También resulta muy destacable el 2 \% de la categoría "No lo consideran necesario".

A través de las propuestas que se están realizando en la Facultad de Ciencias de la Educación que se mostrarán a continuación, se pondrá de manifiesto que la falta de espacio no tiene por qué ser una razón de peso para no tener huerto escolar en un Centro. Resulta de interés contrastar el alto porcentaje de centros encuestados sin huerto y que el principal motivo para no tenerlo sea la falta de espacio, con la baja consideración de innecesaria la instalación de un huerto. Es decir, hay un número elevado de centros escolares con educación infantil en Córdoba que no tienen huerto escolar, la casi totalidad considera interesante tenerlo y no lo tienen en gran parte por parte falta de espacio.

Por otra parte, el $14 \%$ que muestra la categoría "Nadie dispuesto a llevarlo a cabo" puede estar relacionado con el hecho de que la puesta en marcha y el mantenimiento de un huerto requiere de un esfuerzo y una dedicación extra. Para subsanar en parte este inconveniente, y para que el huerto sea una actividad educativa completa, es importante vincular a las familias en el proceso de construcción y desarrollo del mismo.

Las categorías "No se lo han planteado" y "Falta de conocimientos", suman entre ambas un $24 \%$, que puede agruparse en falta de información y de formación en cuanto a esta actividad escolar. Se espera que las propuestas llevadas a cabo en la formación del alumnado de los grados de educación, ayuden a largo plazo a mejorar estos resultados, acercando el huerto escolar y los conocimientos para su desarrollo al futuro profesorado.

Los resultados de la segunda pregunta (¿De qué espacios disponen?) se presentan en el Gráfico 2. 
GRÁFICO 2. Frecuencia relativa, en tanto por ciento, de respuesta de los centros escolares con educación infantil de la ciudad de Córdoba en relación a la pregunta: ¿De qué espacios disponen?

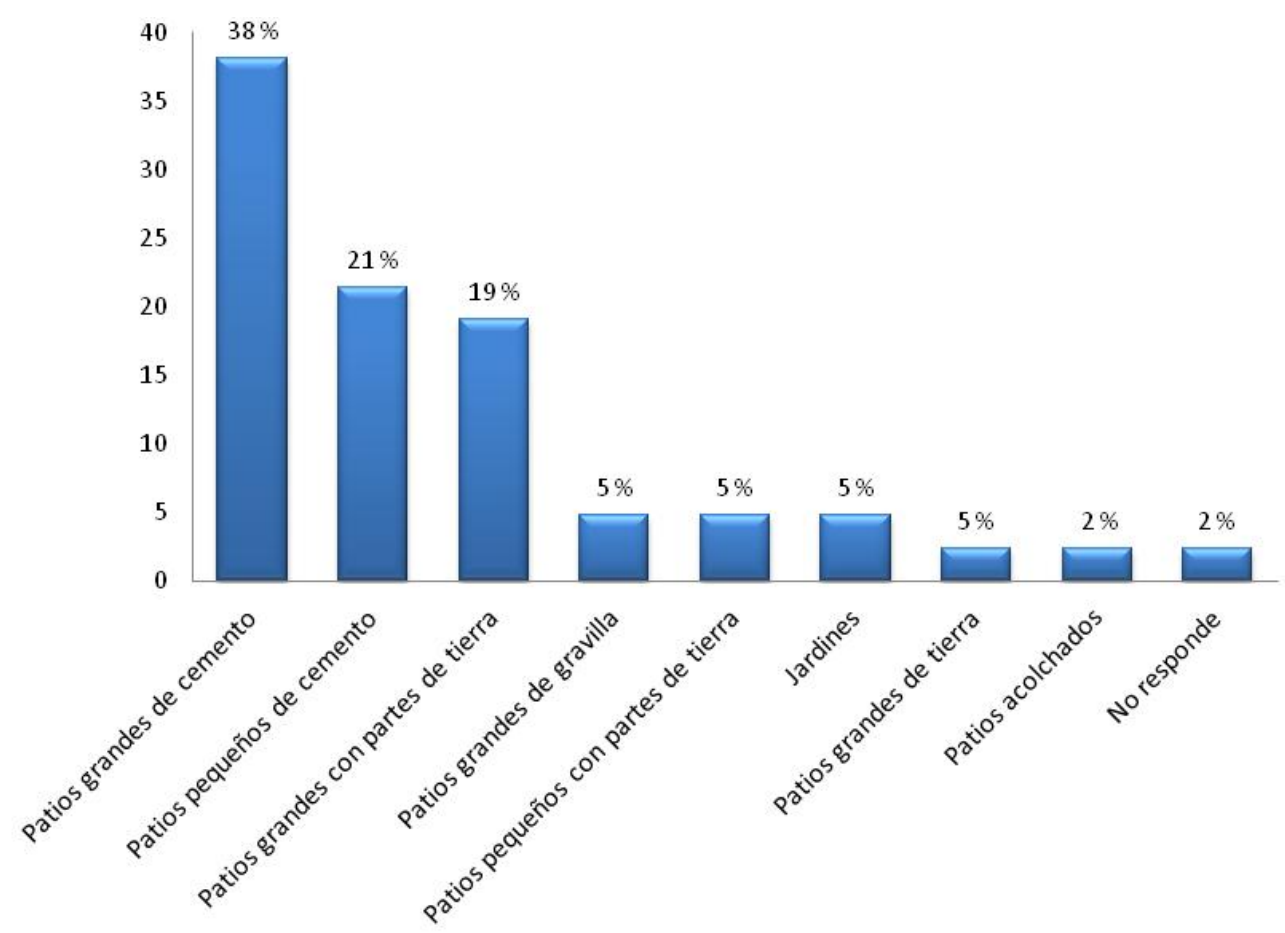

Fuente: Elaboración propia

En esta ocasión se han considerado 9 categorías. De entre ellas, la que ha presentado una frecuencia relativa más alta ha sido "Patios grandes cementados", mostrando una frecuencia cercana al $40 \%$, seguida de "Patios pequeños cementados" con poco más de un $20 \%$. El hecho de que casi el $60 \%$ de los centros posea patios cementados, podría ser una de las causas de que el 51,6 \% de los centros encuestados no posea un huerto escolar, máxime si se desconocen las técnicas que superan el inconveniente de no poseer suelo cultivable. De nuevo, las iniciativas que se mostrarán a continuación permiten subsanar el problema de la ausencia de suelo libre en los centros educativos para la instalación de un huerto escolar.

\subsection{Analizar si en dichos centros se conocen algunas de las técnicas de planificación, diseño e implementación de un huerto escolar}

La tercera y última pregunta ¿Conoce las distintas técnicas para instaurar un huerto en su Centro? ha suscitado los resultados que se detallan a continuación: Se han generado, tan solo, 3 categorías. Destaca el casi 70 \% de la categoría “No", es decir, la mayoría de los colegios encuestados que no poseen huerto, desconocen las técnicas para instaurarlo. Dicho desconocimiento está directamente relacionado con la ausencia de huerto en los mismos. Para realizar esta afirmación nos basamos en que casi la mitad de las personas encuestadas han respondido que es la falta de espacio la razón de no tener huerto escolar en el centro y que en casi el $60 \%$ de los centros el suelo está cementado, y las técnicas de cultivo que a continuación se presentan, permiten solventar ambos inconvenientes. Dichas técnicas se muestran en las prácticas del alumnado de Grado de Educación Infantil, que serán los futuros educadores y educadoras de la etapa de educación infantil. 
5.4. Mostrar las diferentes técnicas de cultivo que posibilitan el desarrollo de esta actividad en cualquier centro a través de las prácticas llevadas a cabo en la Facultad de Ciencias de la Educación de la Universidad de Córdoba como parte de la formación del alumnado de Grado en Educación Infantil

La primera iniciativa que se va a mostrar es el huerto con fines docentes de la Facultad de Ciencias de la Educación. Se trata de la instalación de un huerto no permanente, sin la modificación de la infraestructura del Centro, y de un invernadero portátil con mesas para colocar semilleros. Se muestra así una modalidad de huerto reproducible en cualquier centro escolar que posea un espacio exterior. El material fue adquirido en un centro comercial de la localidad y la factura resultó muy asequible. El precio del mismo se puede abaratar adquiriendo el material a través del contacto con las instituciones que ostentan los servicios de limpieza y reciclaje de la ciudad, tanto para la adquisición de la construcción del huerto como para el mantillo.

En este caso, la instalación y la financiación de la misma se realizaron gracias al Proyecto Coordinado de Innovación de la Facultad de Ciencias de la Educación de la Universidad de Córdoba (convocatoria 2015 y código 2015-1-6001).

La construcción del huerto se realizó durante las prácticas de la asignatura de Didáctica del Medio Ambiente en Educación Primaria en cuatro fases (Imagen 1). (1) Una vez se disponga del material, hay que seleccionar el lugar donde se va a colocar el huerto. (2) Este se compone de un cajón sin fondo realizado con traviesas de madera, fijadas entre ellas con placas y tirafondos. (3) Para aislarlo del suelo y evitar la pérdida del mismo, se colocó una tela aislante de jardinería a modo de forro del cajón. (4) Posteriormente, se llenó el cajón de mantillo. Por último, se ha instalado un sistema de riego por goteo. Si el centro no tuviera una toma de agua cercana, se establecerán turnos de riego a mano.

IMAGEN 1. Construcción del huerto no permanente con fines docentes en la Facultad de Ciencias de la Educación en cuatro fases: 1. Elección de la ubicación; 2. Construcción del cajón; 3. Forrado del cajón con tela aislante; 4. Llenado del cajón con mantillo

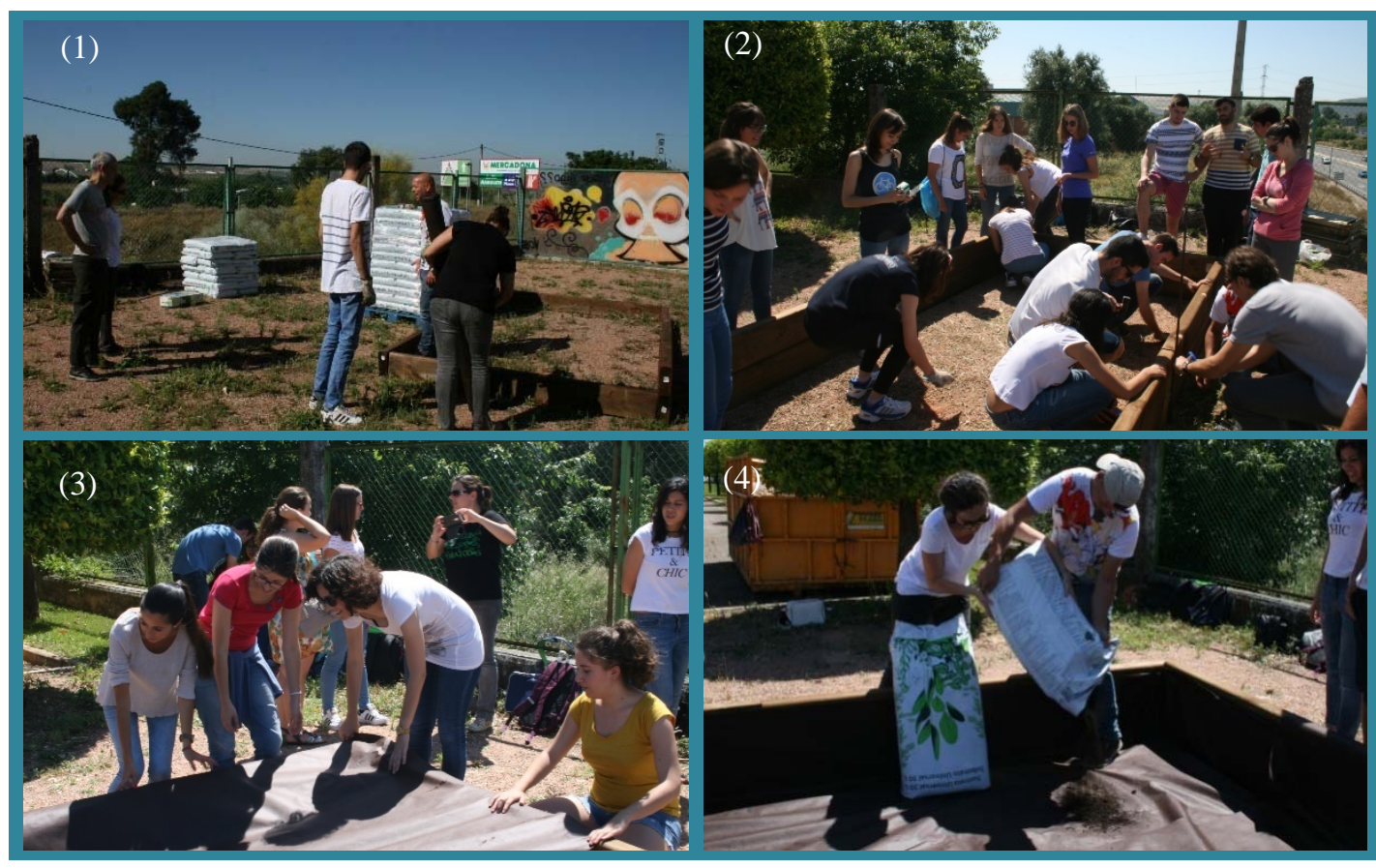

Fuente: Elaboración propia 
También se construyó un semillero en el que se han instalado mesas de siembra (Imagen 2). El invernadero nos permite trabajar todo el proceso con más garantía de éxito, ya que el sembrado directo suele mostrar una producción mucho más baja, por la exposición de las semillas y las plantas a un ambiente menos controlado, susceptible a los cambios de temperatura y a los animales.

IMAGEN 2. Invernadero con mesas para la siembra y semilleros

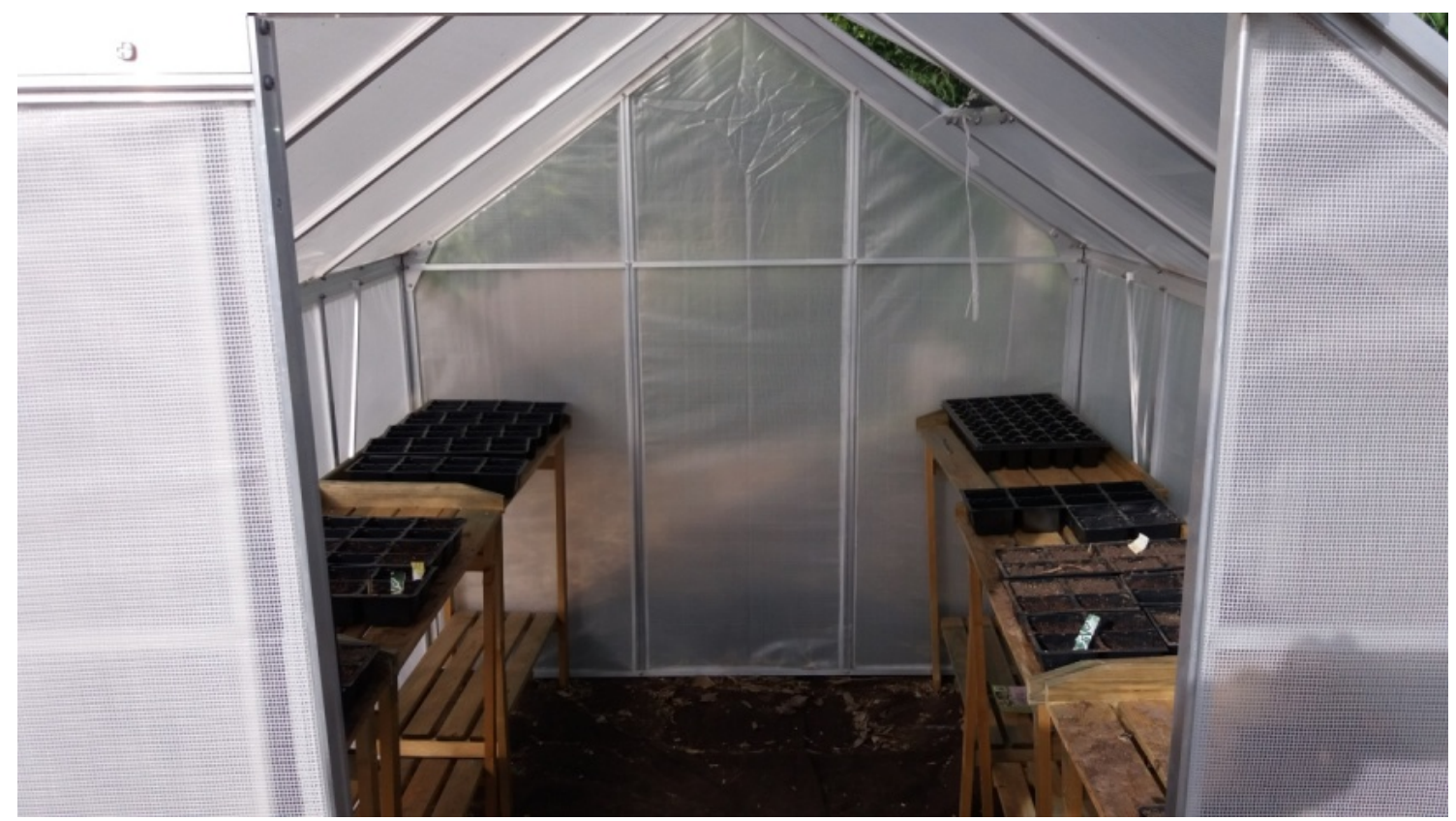

Fuente: Elaboración propia

El invernadero y el huerto escolar se utilizan cada curso desde su instalación en las prácticas de la asignatura Didáctica de las Ciencias Naturales y Sociales en Educación Infantil de tercero de Grado de Educación Infantil. En primer lugar, se muestra el proceso de construcción de ambos. También se reflexiona sobre las posibilidades de uso del huerto en la docencia en Educación Infantil:

- Las plantas son seres vivos.

- Ciclo de vida de las plantas.

- Necesidades de las plantas.

- El huerto como ecosistema: relaciones entre seres vivos y seres inertes, y relaciones de los seres vivos entre sí.

- La importancia de la agricultura ecológica en la sostenibilidad del planeta.

Ya en dichas instalaciones el alumnado realiza las labores de limpieza del huerto, siembra en semilleros, trasplante de plantones, etc., para la producción de flores y hortalizas (Imagen 3). Cada persona tiene asignado un semillero y un plantón. Todas las actividades descritas se realizan en una sesión de prácticas. 
IMAGEN 3. Vista del invernadero y el huerto con fines docentes de la Facultad de Ciencias de la Educación de la Universidad de Córdoba en funcionamiento

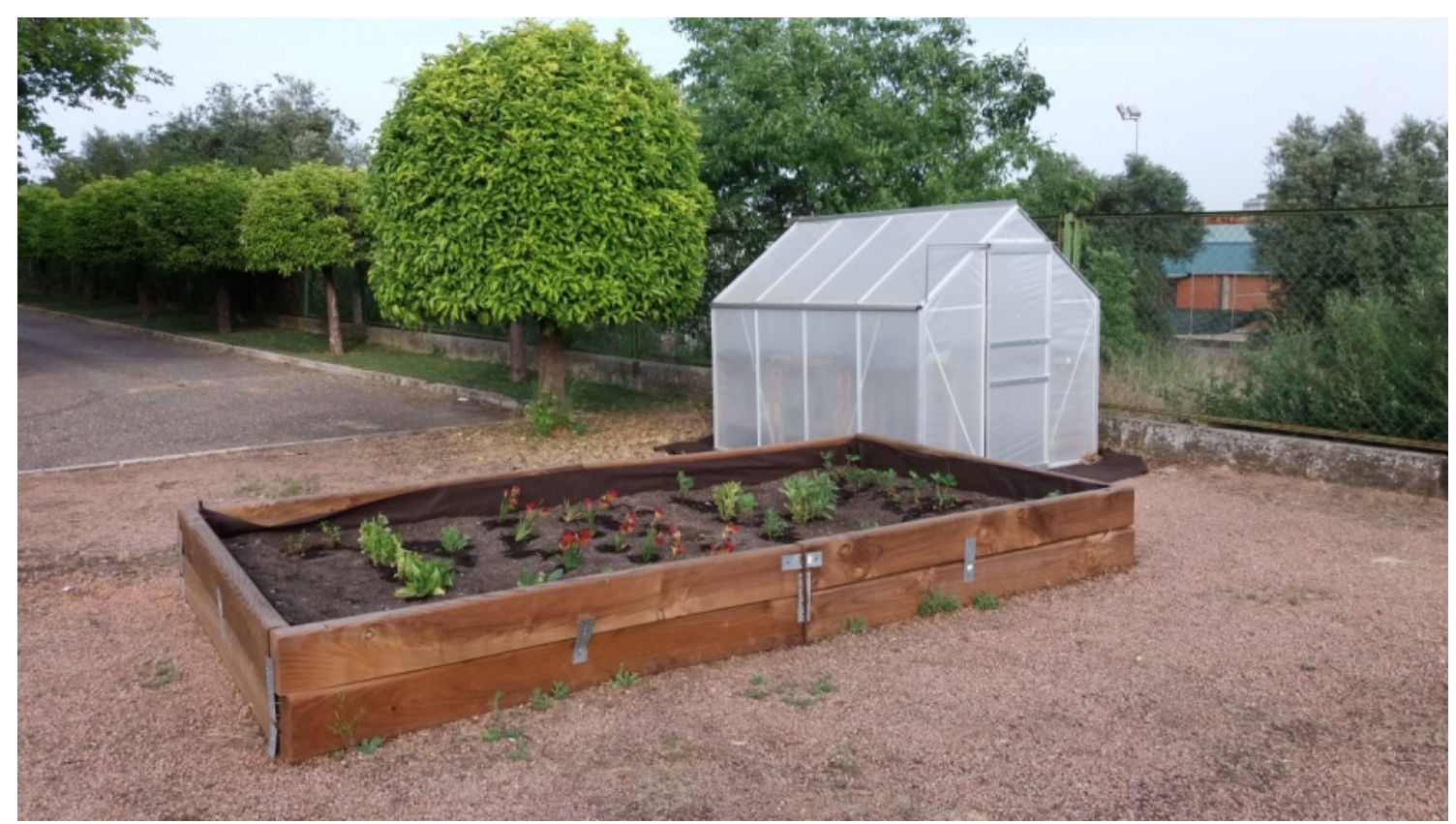

Fuente: Elaboración propia

Fuera del horario lectivo, el alumnado tiene que realizar el cuidado y el seguimiento semanal tanto de la germinación de las semillas sembradas, como del crecimiento de los plantones. Para la evaluación de la práctica, el alumnado tendrá que entregar un dossier con una descripción de las labores y del seguimiento realizado, con al menos dos gráficas, una relativa a la cantidad de brotes por semana, y la otra en relación al crecimiento semanal de los plantones. Para finalizar, tendrán que incluir una propuesta de actividades para trabajar el huerto en educación infantil.

La fabricación de un huerto para este ciclo educativo no tiene por qué emplear grandes extensiones ni materiales costosos, ya que podemos llevar la misma iniciativa en un formato más reducido, usando para ello cajas de siembra y cultivo. Este es el leitmotiv que inspira la segunda iniciativa, que se lleva a cabo desde la asignatura Educación para la Salud y el Consumo, optativa del cuarto curso del Grado de Infantil. En esta asignatura, se incide en los aspectos más problemáticos de la promoción de la salud, así como en la idoneidad de promover hábitos de consumo responsable en las etapas iniciales de la educación. Como trabajo grupal, se insta al alumnado de la asignatura a que, durante el transcurso de la misma, elaboren y cuiden un huerto realizado en una caja, con hortalizas estacionales. Esta práctica, que tiene un peso del $20 \%$ en la nota final de la asignatura, supone un reto para estos futuros docentes de Educación Infantil, que deben cuidar, cada semana un alumno o alumna del grupo, un sistema vivo que requiere unas pequeñas pautas de riego, temperatura y luminosidad.

La práctica se desarrolla la primera semana donde grupos de 4-5 alumnos eligen qué hortalizas plantar y realizan la siembra, siendo este primer día el punto de partida del cuidado del huerto realizado (Imagen 4). Antes de iniciar la práctica, se les realiza una pequeña exposición teórica sobre la misma, incidiendo en los aspectos a controlar para el correcto desarrollo del huerto, así como las pautas de la exposición final, que es la última práctica de la asignatura y supone la recogida de los resultados de cada grupo. 
IMAGEN 4. Creación del huerto portátil en cajas de siembra en la asignatura de Educación para la Salud y el consumo

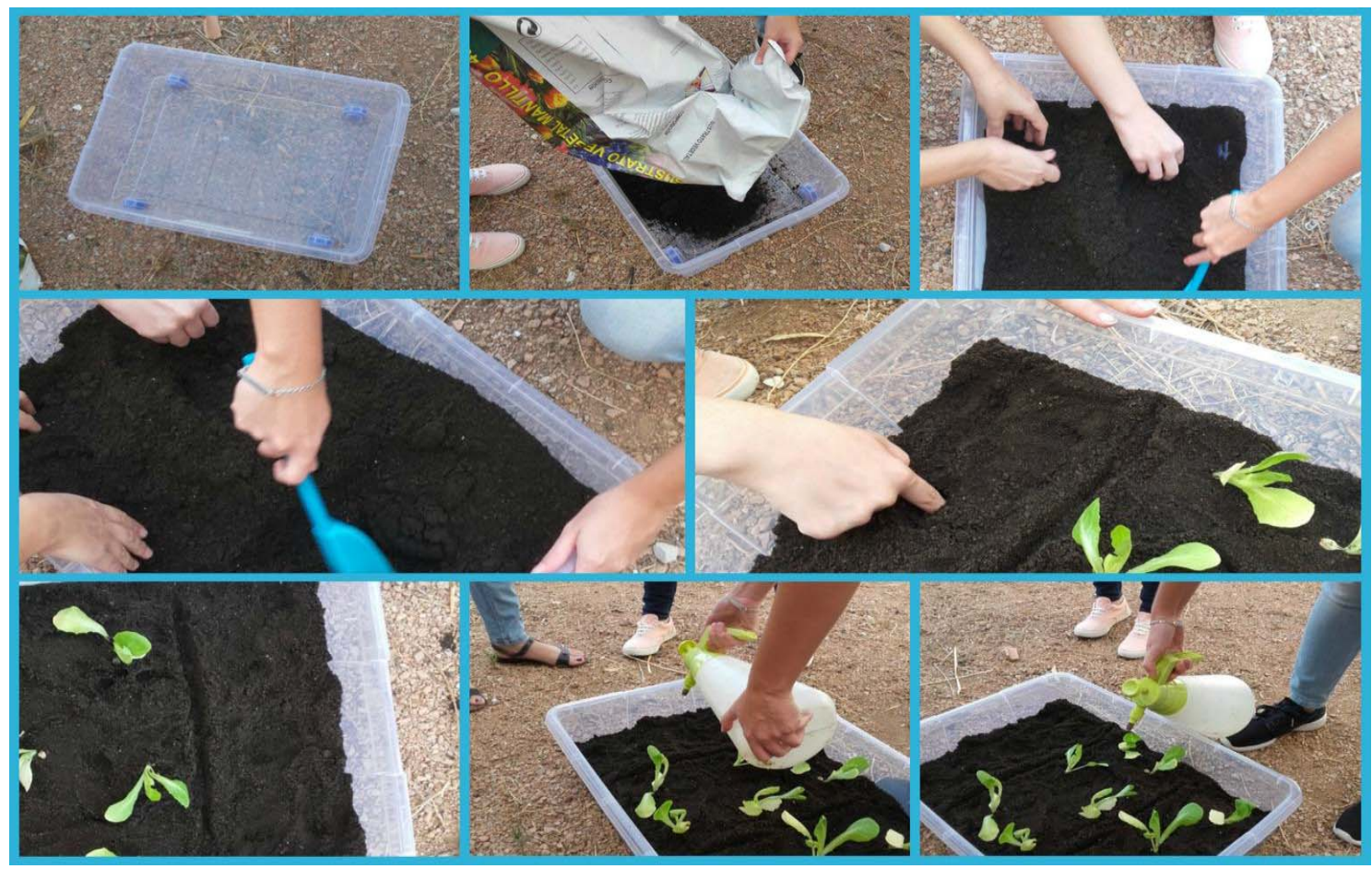

Fuente: Elaboración propia

Como es una asignatura cuatrimestral, estos huertos creados deberán cuidarse un mínimo de 3 meses, siendo imprescindible la toma de anotaciones, así como de registro gráfico de los progresos. Durante este tiempo, el alumnado puede preguntar las dudas generadas al profesorado de la asignatura, siendo importante la toma de decisiones ante las posibles problemáticas.

La última práctica de la asignatura consiste en una exposición donde cada grupo debe resaltar cómo ha sido la evolución de su huerto y qué problemas han encontrado durante el tiempo de desarrollo del mismo. El apartado expositivo de mayor puntuación respondía a la pregunta A la vista de los resultados obtenidos, ¿cuáles son tus propuestas para llevar esta iniciativa al ciclo de Educación Infantil?, donde el alumnado debía proponer ideas innovadoras y pautas de trabajo que repercutieran en la promoción de hábitos saludables de consumo responsable. En este sentido, algunas de las ideas planteadas pasaban por repartir roles de trabajo en el alumnado de infantil, implicar la participación de la familia en las actividades generadas (que podrían pasar desde las propias del cuidado del huerto realizado - en el formato más idóneo para tenerlo en clase, ya fuera latas, cajas de siembra, etc. - hasta las que implicaban una reflexión de estos niños y niñas - que pasaban por adivinanzas, cuentos con moraleja, búsqueda de información de las hortalizas plantadas, etc.).

El alumnado se ha mostrado muy interesado durante el desarrollo de las prácticas descritas y han alcanzado una elevada calidad en sus trabajos relativos a las mismas. A continuación se recogen algunas de sus opiniones:

Jennifer: "Creo que la experiencia de trabajar en un huerto ha sido interesante y se puede emplear como un recurso más para acercar a los niños y a las niñas de Educación Infantil a la naturaleza, e implicarlos en el cuidado y respeto de esta, además de inculcarles valores como la responsabilidad, el trabajo en equipo y la cooperación.” 
Sandra: "Gracias a esto he podido experimentar personalmente el huerto y ahora, como futura docente, estoy segura de que cuando tenga mi propia clase practicaré esta experiencia con mis alumnos.”

Alba: "Para concluir me gustaría destacar que considero la actividad realizada como una dinámica muy importante para trabajarla con los niños de infantil, ya que siguiendo su proceso y a través de la experiencia pueden ser conscientes de todo lo que necesita una planta para vivir, sus propiedades, sus usos y su crecimiento de una manera más llamativa, lúdica y motivadora.”

Trabajar al aire libre y en contacto con la naturaleza es algo necesario, y más en las primeras etapas, por lo que el huerto escolar es un recurso adecuado para este propósito (Bundschu-Mooney, 2003; Mérida Serrano, Torres-Porras y Alcántara Manzanares, 2017). Aunque esto no quiere decir que en los centros sin huerto no se trabaje la naturaleza, ya que existen otras metodologías de acercamiento a la misma (Amórtegui Cedeno, Mayoral García-Berlanga y Gavidia Catalán, 2017), para todas las etapas educativas desde la educación infantil hasta la educación superior, como salidas a entornos naturales como parques urbanos (Torres-Porras, Alcántara, Arrebola, Rubio y Mora, 2017), los itinerarios didácticos (Alcántara Manzanares, Rubio, Mora, 2014), etc.

Trabajar el huerto en la escuela puede permitir pasar tiempo al aire libre, trabajar manualmente, observar elementos naturales y desencadena una serie de beneficios en el alumnado como apreciar más los vegetales como alimento (Langelloto y Gupta, 2012), influir en las actitudes ambientales (Skelli y Zajicek, 1998), así como propiciar las interacciones sociales y habilidades personales creando a su vez una actitud más positiva hacia el colegio (Waliczek, Bradley y Zajicek 2001). Promover hábitos de consumo responsable y de alimentación saludable pasa, en definitiva, por conocer los alimentos, desde la huerta a la mesa, y su producción sostenible.

Con las dos iniciativas desarrolladas en la Facultad de Ciencias de la Educación de la Universidad de Córdoba se pretende que el futuro profesorado conozca el huerto escolar, sus virtudes como recurso educativo y las técnicas que permitan superar los problemas que impiden en la actualidad su implantación en los centros escolares con Educación Infantil.

\section{CONCLUSIONES}

Este artículo muestra que los huertos escolares no son una realidad educativa en la mayoría de centros educativos de la etapa de educación infantil en la ciudad de Córdoba, siendo las principales causas argumentadas para no poseer un huerto: la falta de espacio; la ausencia de suelo cultivable y el desconocimiento de las técnicas alternativas de instalación de un huerto escolar.

Es imprescindible actuar para provocar el cambio de esta situación, ya que existen múltiples opciones para que cualquier centro educativo posea un huerto. Este cambio puede iniciarse en la universidad, en los grados de educación, tal y como se muestra en este trabajo. Fomentando el conocimiento de las técnicas necesarias para instaurar el huerto escolar, motivando al alumnado universitario de los grados de educación a participar de este recurso. Así se puede conseguir que dicho alumnado conozca y pueda poner en práctica en un futuro en sus centros educativos el huerto escolar para que pase de ser una innovación educativa a una realidad en las escuelas.

Una cuestión de gran interés y que se abordará en futuras investigaciones es conocer el uso que se está realizando del huerto escolar en aquellos centros que lo han implementado. Dicho conocimiento nos permitirá tener un retrato completo del grado de implantación del huerto escolar, así como identificar buenas prácticas y deficiencias de uso, que podrían conllevar acciones formativas, relacionadas con este recurso, para el citado alumnado universitario.

Las innovaciones educativas necesitan apoyarse en los resultados de una investigación previa que justifique y guíe su implementación, del mismo modo que requieren de un análisis posterior para identificar si los esfuerzos en convertirlas en realidades educativas son suficientes o hay que perseverar. Así, los huertos no son una realidad educativa de los centros de educación infantil de la ciudad de Córdoba, ya que no se presentan en la mayoría de los centros, y su ausencia se argumenta 
sobre todo en la falta de espacio. Para apoyar a que los huertos sean una realidad educativa, se han presentado una serie de propuestas formativas, en relación a las diversas formas de implantación de un huerto en la escuela, sea cual sea su disponibilidad de espacio o las características de espacios libres de la misma, para el alumnado de Grado de Educación Infantil. Se espera que al trabajar en la formación inicial del profesorado, se incida en la mejora educativa, y en este caso, el uso del huerto escolar sea mayoritario.

\section{Referencias}

Alcántara Manzanares, J., Rubio, S.J., Mora, M. (2014). Google Earth como herramienta para formadores en la preparación de itinerarios didácticos. En R. Martínez Medina y E. M. Tonda-Monllor (Coords.), Nuevas perspectivas conceptuales y metodológicas para la educación geográfica(pp. 47-53). Madrid, España: Grupo de Didáctica de la Geografía de la Asociación de Geógrafos Españoles (AGE).

Amórtegui Cedeno, E.F., Mayoral García-Berlanga, O., y Gavidia Catalán, V. (2017). Aportaciones de las Prácticas de Campo en la formación del profesorado de Biología: un problema de investigación y una revisión documental. Didáctica de las Ciencias Experimentales y Sociales, 32, 153-169. DOI: 10.7203/dces.32.9940.

Aranceta, J. (2008). Realidad actual de los comedores escolares en España. En J. R. Martínez y I. Polanco. (Coords.), El libro blanco de la alimentación escolar (pp. 1-12). Madrid: McGrawHill.

Barrón Ruiz, A. y Muñoz Rodríguez, J.M. (2015). Los huertos escolares comunitarios: fraguando espacios socioeducativos en y para la sostenibilidad. Foro de Educación. DOI: 2015.013.019.010.

Botella, A. M., Hurtado, A. y Cantó, J. (2014). Las competencias básicas a través del huerto escolar: una propuesta de proyecto de innovación. En J. J. Maquilón Sánchez y N. Orcajada Sánchez (Eds.). Investigación e innovación en formación del profesorado (pp. 173-182). Murcia: Servicio publicaciones Universidad de Murcia (Edit.um).

Brantsæter, A.L., Ydersbond, T.A., Hoppin, J.A., Haugen, M. y Meltzer, H.M. (2017). Organic Food in the Diet: Exposure and Health Implications. Annual Review of Public Health, 38, 295-313. DOI: 10.1146/annurev-publhealth-031816-044437.

Bueno, M. (2011). Salud-Hábitat-Conciencia. Recuperado de http://www.marianobueno.com/2011/12/07/el-huerto-ecologico-cultiva-tu-salud-y-la-del-planeta/ [17 de febrero de 2016].

Bundschu-Mooney, E. (2003). School garden investigation: Environmental awareness and education. San Rafael, CA: Division of Education, School of Business, Education and Leadership, Dominican University of California. (ERIC Document Reproduction Service No. ED480981).

Ceballos, M. (2017). Aprovechamiento didáctico de los huertos escolares en centros de Sevilla. Enseñanza de las ciencias, núm. Extra 2017, 787-792. Recuperado de https://ddd.uab.cat/ record/184560 [28 de abril de 2018]

Chawla L., y Cushing D. (2007). Education for strategic environmental behavior. Environmental Education Research, 13, 437-452.

Collado S. y Corraliza J. A. (2011). Children's perceived restoration and pro-environmental beliefs. Journal of Asian Behavioural Studies, 2, 1-10.

Corraliza J. A., Collado S. y Bethelmy L. (2012). Nature as a Moderator of Stress in Urban Children. Procedia - Social and Behavioral Sciences, 38, 253-263.

Davis B., Rea T. y Waite S. (2006). The special nature of outdoors: Its contribution to the education of children at aged 3-11. Australian Journal of Outdoor Education, 10, 3-12. 
De los Ríos-Carmenado, I., Becerril-Hernández, H. y Rivera, M. (2016). La agricultura ecológica y su influencia en la prosperidad rural: visión desde una sociedad agraria (Murcia, España). Agrociencia, 50(3), 375-389.

El-Hage, N. y Hattam, C. (2003). Agricultura orgánica, ambiente y seguridad alimentaria. Roma: FAO.

Escutia, M. (2009). El huerto escolar ecológico. Barcelona, España: Graó.

Faber Taylor A. y Kuo F. E. (2009). Children with attention deficits concentrate better after walk in the park. Journal of Attention Disorders, 12(5), 402-409.

Flórez, J. (2009). Agricultura ecológica: manual y guía didáctica. Madrid: Ediciones Mundi Prensa.

Goodall, J. (2007). Otra manera de vivir. Cuando la comida importa. Barcelona, España: Lumen.

Grupo de Desarrollo Rural de la Campiña y los Alcores. (s.f.). Red de Ecohuertos escolares. Recuperado de http://www.campialcores.org/medio_ambiente/index.php?RED_DE ECOHUERTOS_ESCOLARES\%26nbsp\%3B [6 de abril de 2016]

Hidalgo, M.I. y Güemes, M. (2011). Nutrición del preescolar, escolar y adolescente. Pediatría integral, 15 (4), 351-368. Recuperado de http://www.pediatriaintegral.es/wpcontent/uploads/2012/03/Pediatria-Integral-XV-4.pdf\#page=52 [12 de febrero de 2016]

Izquierdo, A., Armenteros, M., Lancés, L. y Martín, I. (2004). Alimentación saludable. Revista Cubana de Enfermería, 20(1), 1-1. Recuperado de http://scielo.sld.cu/scielo.php?script= sci_arttext\&pid=S0864-03192004000100012 [11 de febrero de 2016].

Kauffman, M. (1995). Huertos, y más huertos. Investigando en la escuela infantil. Investigación en la escuela $25,87-100$.

Langellotto, G. A. y Gupta, A. (2012). Gardening increases vegetable consumption in school-aged children: A meta-analytical synthesis. HortTechnology, 22(4), 430-445.

Larrubia Vargas, R., Natera Rivas, J.J. y Navarro Rodríguez (2017). La producción ecológica como estrategia de competitividad en los mercados saturados. Estructura productiva de las explotaciones en Andalucía. Boletín de la Asociación de Geógrafos Españoles, 71, 151-177.

Mérida Serrano, R., Torres-Porras, J., y Alcántara Manzanares, J. (2017). Didáctica de las Ciencias Experimentales en Educación Infantil. Madrid: Síntesis.

Miralpeix, A. (2003). La alimentación infantil. Barcelona: Plaza y Janés.

Muehlhoff, E. (2006). Crear y manejar un huerto escolar. Un manual para profesores, padres y comunidades. Roma: FAO.

Orden de 5 de agosto de 2008, por la que se desarrolla el Currículo correspondiente a la Educación Infantil en Andalucía. Boletín Oficial de la Junta de Andalucía. Núm 169. Sevilla, 26 de agosto de 2008. Recuperado de http://www.juntadeandalucia.es/boja/2008/169/3 [11 de marzo de 2016]

Ozdemir A. y Yilmaz O. (2008). Assessment of outdoor school environments and physical activity in Ankara's primary schools. Journal of Environmental Psychology, 28, 287-300.

Ratcliffe, M. M., Merrigan, K. A., Rogers, B. L. y Goldberg, J. P. (2011). The effects of school garden experiences on middle school-aged students' knowledge, attitudes, and behaviors associated with vegetable consumption. Health Promotion Practice, 12, 36-43.

Remmers, G. (1993). Agricultura tradicional y agricultura ecológica: vecinos distantes. Agricultura y sociedad, (66), 201-220. Recuperado de http://www.magrama.gob.es/ministerio/pags/ biblioteca/revistas/pdf_ays/a066_07.pdf [4 de febrero de 2016]

Silva, C. (2007). Trastornos alimentarios. México: Ed. Pax L.C.C., S.A.

Skelly, S. M. y Zajicek, J. M. (1998). The effect of an interdisciplinary garden program on the environmental attitudes of elementary school students. HortTechnology, 8(4), 579-583.

Torres-Porras, J., Alcántara, J., Arrebola J.C., Rubio, S.J. y Mora, M. (2017). Trabajando el acercamiento a la naturaleza de los niños y las niñas en el Grado de Educación Infantil. 
Crucial en la sociedad actual. Revista Eureka sobre Enseñanza y Divulgación de las Ciencias, 14(1), 258-270..

Waliczek, T. M., Bradley, J. C., y Zajicek, J. M. (2001). The effect of school gardens on children's interpersonal relationships and attitudes toward school. HortTechnology, 11(3), 466-468.

Wells N. M. (2000). At home with nature: The effects of nearby nature on children's cognitive functioning. Environment and Behaviour, 32, 775-795.

Wolch J., Jerrett M., Reynolds K., McConnell R., Chang R., Dahmann N., Brady K., Gilliland F., Su J.G. y Berhane K. (2011). Childhood obesity and proximity to urban parks and recreational resources: A longitudinal cohort study. Health \& Place, 17, 207-214.

\section{CÓMO CITAR ESTE ARTÍCULO}

Alcántara, J., Torres-Porras, J., Mora, M., Rubio, S. J., Arrebola, J. C. y Rodríguez, L. (2019). ¿Son los huertos escolares en educación infantil una realidad o una innovación educativa? Estudio de centros escolares de la ciudad de Córdoba (España) y propuestas de cambio desde la Universidad. Didáctica de las ciencias experimentales y sociales, 36, 79-96. DOI: 10.7203/DCES.36.12535. 
